\title{
Quantum-dots-dispersed bent-core nematic liquid crystal and cybotactic clusters: Experimental and theoretical insights
}

\author{
Sourav Patranabish, ${ }^{1}$ Yiwei Wang, ${ }^{2}$ Aloka Sinha, ${ }^{1}$ and Apala Majumdar ${ }^{3}$ \\ ${ }^{1}$ Department of Physics, Indian Institute of Technology Delhi, Hauz Khas, New Delhi 110016, India \\ ${ }^{2}$ Department of Applied Mathematics, Illinois Institute of Technology, Chicago, Illinois 60616, USA \\ ${ }^{3}$ Department of Mathematics and Statistics, University of Strathclyde, Glasgow G11XQ, United Kingdom
}

(Received 16 August 2020; revised 6 April 2021; accepted 9 April 2021; published 14 May 2021)

\begin{abstract}
We study a quantum-dots (QDs) dispersed bent-core nematic liquid crystalline system in planar geometry and present experimental measurements of the birefringence $(\Delta n)$, order parameter $(S)$, dielectric dispersion, absorption spectra, and optical textures with attention to variations with temperature. A bent-core liquid crystal (LC) $14-2 \mathrm{M}-\mathrm{CH}_{3}$ is used as the host material and $\mathrm{CdSe} / \mathrm{ZnS}$ core-shell type QDs are used as the dopant. The nematic $(N)$ phase of the pristine (undoped) $\mathrm{LC} 14-2 \mathrm{M}-\mathrm{CH}_{3}$ contains cybotactic clusters, which are retained by its QDs incorporated LC nanocomposite. Our experimental findings support: (i) reduced orientational order parameter of the QDs dispersed LC system compared to its pristine counterpart at fixed temperatures, (ii) reduced cybotactic cluster sizes due to the incorporation of QDs, and (iii) increased activation energies related to reduced cluster sizes. We complement the experiments with a novel Landau-de Gennes-type free energy for a doped bent-core LC system that qualitatively captures the doping-induced reduced order parameter and its dependence on the properties of the QDs and its variation with temperature.
\end{abstract}

DOI: 10.1103/PhysRevE.103.052703

\section{INTRODUCTION}

The bent-core liquid crystals (BLCs) are a novel class of liquid crystal (LC) mesogens that manifest various unique and exciting properties, such as chirality, ferroelectricity, and biaxiality [1-6]. They are known to form several exotic mesophases, such as the twist-bend nematic $\left(N_{\mathrm{tb}}\right)$ phase, the blue phase, and the banana (B1-B7) phases [2,7-9]. The nematic $(N)$ phase of BLCs itself manifests a few of the aforementioned distinct features, such as ferroelectric response, fast switching, and macroscopic biaxiality [1,4,10-13]. The main reason behind these extraordinary features is the locally polar cybotactic clusters formed by BLC molecules in their $N$ phase [4-6,10-13]. Due to bent molecular shape and the lack of translational symmetry, the BLC molecules in their $N$ phase experience steric hindrance. This causes stacking of the BLC molecules in smectic layers (clusters) [14,15]. These stacks of molecules are termed as "cybotactic" clusters because they are more ordered compared to the surrounding molecules. The clusters and the other BLC molecules together constitute the macroscopic $N$ phase [4]. Recent reports, aided by various experimental techniques, have established the existence of cybotactic clusters in the nematic, smectic, and even in the isotropic phases [12,16-20]. Although studied extensively, the origins of cluster formation and the effects of external factors (e.g., nanoparticle doping, electric field) on these clusters remain an open problem. Further studies are required for the manipulation and successful tailoring of cybotactic clusters for applications in science and technology, including novel BLC-driven devices.

Suspension of nanoparticles (NPs) in the LC matrix to improve or to selectively modify the physical properties of
LCs is a widely used technique in today's liquid crystal science. Studies have shown that the dispersion of nanoparticles in LCs can improve the electro-optic properties, modify the elastic anisotropy and the dielectric constants, and reduce the transition temperatures [21-25]. The incorporation of NPs can also affect the orientation of LCs and induce a homeotropic alignment [26]. Varying the size and shapes of the dopant NPs also have a profound effect on the physical properties of LCs [27-29]. Recently, a new class of semiconductor NPs have been discovered, called the quantum dots (QDs). Incorporation of these QDs in the LC matrix may also affect or alter the physical properties of LCs, such as a reduction in the dielectric anisotropy, faster response times, changes in the phase transition temperatures, and altered boundary conditions [25,28-32]. Changes in the dielectric anisotropy $(\Delta \epsilon)$ provide an indirect measure of changes in the order parameter $(S)$ because $\Delta \epsilon \propto S[25,33]$. The QDs are usually capped with functionalized ligands that prevent aggregation. In particular, this makes QDs good candidates for stabilizing dilute suspensions in doping or dispersion LC experiments. To date, there has been work on QDs dispersed in calamitic nematic LCs (NLCs) whereas their effect on bent-core NLCs is relatively open [25]. In particular, little is known about the effect of QDs or doping, in general, on the cybotactic clusters in bent-core NLCs and in the absence of systematic experimental and theoretical studies on these lines, doped bent-core NLC systems cannot meet their full potential.

We study a dilute homogeneous suspension of a QDdoped thermotropic BLC (details in the next section), confined in a planar cell with fixed boundary conditions on both cell surfaces. In particular, the undoped counterpart exhibits cybotactic clusters. Our primary investigations concern 
comparisons between the doped and the undoped systems that give quantitative and qualitative insight into the effects of doping, the interplay between doping and cluster formation, and how these effects can be tailored by temperature and external stimuli. This paper builds on our first paper [34] wherein we focused on a one-dimensional theoretical study of the $N$ phase of a BLC, confined in a planar cell, within a phenomenological Landau-de Gennes (LdG) framework inspired by previous insightful modeling in Ref. [35]. This model is based on the premise that the $N$ phase of BLC is characterized by two order parameters: $S_{g}$ that measures the ordering of the ground-state molecules (outside the clusters) and $S_{c}$ that measures the ordering within the smecticlike cybotactic clusters with coupling between the two effects captured by an empirical parameter $\gamma$. In Ref. [34], we theoretically study the effects of spatial inhomogeneities, confinement, and the coupling parameter $\gamma$ on $S_{g}$ and $S_{c}$. Little is known about the material-dependent values of $\gamma$ or indeed how it could be experimentally controlled. Our theoretical studies showed that larger values of $\gamma$ substantially increase the interior values of $S_{g}$ and $S_{c}$, i.e., $\gamma$ promotes order outside and within the clusters of the $N$ phase of the BLC, the effects being more pronounced for lower temperatures. However, the coupling also enhances the values of $S_{g}$, for temperatures above the nematic-isotropic transition temperature, i.e., the bent-core NLC can exhibit nematic order when the calamitic $N$ phase does not, e.g., for temperatures above the nematic-isotropic transition temperature. The model in Ref. [34] is simplified in many ways but yet sheds qualitative insight into the powerful prospects offered by cybotactic clusters in BLCs and how they can be used to manipulate nematic order and phase transitions for tailor-made applications.

In this paper, we report a combined experimental and theoretical analysis of a QDs dispersed bent-core nematic LC $14-2 \mathrm{M}-\mathrm{CH}_{3}$ in the dilute regime. The dilute regime applies to systems of nanoscale QDs (much smaller than the system size) with a low concentration of QDs, and the QDs are uniformly dispersed without any aggregation effects. We perform optical texture observations, dielectric measurements, optical birefringence measurements, and the orientational order parameter calculations on the pristine BLC and its QD-dispersed counterpart. The $N$ phase of $14-2 \mathrm{M}-\mathrm{CH}_{3}$ contains cybotactic clusters as already reported in our earlier work [36]. We find that the $N$ phase of the QD-dispersed counterpart also contains cybotactic clusters, albeit with modified properties. We report a number of interesting experimental results for the QD-dispersed BLC system-the optical birefringence $(\Delta n)$ is lowered and the macroscopic order parameter $(S)$ is reduced compared to the undoped counterpart for a given temperature, the activation energy $\left(E_{a}\right)$ increases compared to the undoped counterpart and based on the measurements of the relaxation frequencies $\left(f_{R}\right)$ and activation energies, we deduce that the size of the cybotactic clusters decreases with QDs doping. We complement our experiments with a theoretical LdG-type model for the $N$ phase of the QD-doped BLC, using the framework developed in Ref. [37]. This framework is not specific to QDs or to BLCs but to generic dilute doped LC systems and effectively captures the effects of the homogeneously suspended inclusions (in this case QDs) in terms of an additional contribution to the free energy. Hence, we apply this approach to the LdG free energy of a BLC system proposed in Refs. [34,35] and qualitatively capture the effects of the QDs by means of suitable novel additional energetic terms. These additional terms, in principle, depend on the properties of the QDs, e.g., size, shape, anchoring, and preferred order, etc. We introduce a weighted mean scalar order parameter $S_{m}$, the theoretical analog of the experimentally measured scalar order parameter. This simplistic approach does capture the doping-induced reduction in the mean order parameter $S_{m}$, which, in turn, qualitatively explains the reduction in birefringence, dielectric anisotropy. We present our experimental results in three parts below, followed by the mathematical model, numerical results, and perspectives for future work.

\section{EXPERIMENT}

A thermotropic bent-core nematic LC $14-2 \mathrm{M}-\mathrm{CH}_{3}$ was used in the experiments and as the host for the studied LC nanocomposite. The LC material was obtained from Professor Rao's group at the Department of Chemistry, Assam University, Silchar, Assam, India. The molecular formula of 14-2M- $\mathrm{CH}_{3}$, synthetic scheme details, etc., are available in our earlier paper [36]. The $\mathrm{CdSe} / \mathrm{ZnS}$ core-shell-type QDs of diameter $5.6 \mathrm{~nm}$ (core diameter: $2.8 \mathrm{~nm}+$ shell thickness $1.4 \mathrm{~nm}$ ) were procured from Sigma-Aldrich, Merck (USA) for preparing the LC nanocomposites. The spherical QDs were stabilized with the encapsulation of octadecylamine ligands, and they have absorption maxima in the range from 510 to $540 \mathrm{~nm}$ and emission wavelengths lying in the range of 530$540 \mathrm{~nm}$ as provided by the manufacturer. The sequence of performed experimental steps are as follows: preparation of QDs dispersed LC nanocomposite, optical texture observation, evaluation of transition temperatures, orientational order parameter determination via optical birefringence measurements, and dielectric characterization. All the experimental measurements were carried out while slowly cooling the sample from the isotropic liquid.

To prepare the LC nanocomposite, CdSe/ZnS QDs were taken at $0.5 \mathrm{wt} \%$ concentration and mixed with the LC compound $14-2 \mathrm{M}-\mathrm{CH}_{3}$. To obtain a homogeneous dispersion of the quantum dots in the LC matrix, chloroform was added to the mixture, and the mixture was ultrasonicated until a visibly homogeneous dispersion was achieved (Fig. 1). The mixture was kept at $\sim 60{ }^{\circ} \mathrm{C}$ for 2 to $3 \mathrm{~h}$, and it was then left overnight at room temperature for the slow evaporation of chloroform [38]. Once the chloroform was completely evaporated, $0.5 \mathrm{wt} \%$ QDs dispersed LC nanocomposites were obtained. They were checked visually through a polarizing optical microscope several times, but no aggregation of QDs was noted.

Indium tin oxide (ITO) coated 5- $\mu$ m planar (homogeneous alignment) cells (Instec Inc., USA) were used for the experiments. Two different cells of this type, were used for the pristine LC and the LC nanocomposite, respectively. The LCs were filled in the cells via capillary action around $10^{\circ} \mathrm{C}$ above the clearing temperature. During measurements, the cells were kept inside an Instec HCS302 hot stage, and the temperature was maintained using an Instec MK1000 temperature controller with an accuracy of $\pm 0.01{ }^{\circ} \mathrm{C}$. The liquid crystalline textures were recorded using an OLYMPUS BX-51P 


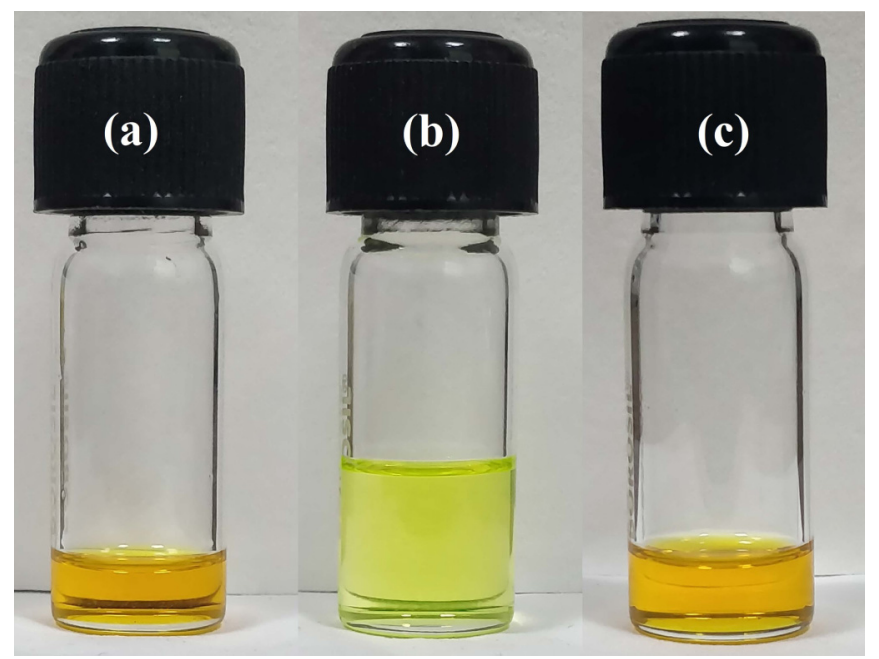

FIG. 1. Visibly homogeneous solutions of (a) $14-2 \mathrm{M}-\mathrm{CH}_{3}$, (b) $\mathrm{CdSe} / \mathrm{ZnS}$ QDs, and (c) nanocomposite (14-2M- $\mathrm{CH}_{3}+$ 0.5 wt \% CdSe/ZnS QD) in chloroform $\left(\mathrm{CHCl}_{3}\right)$.

polarizing optical microscope (POM) attached to a computer with the sample placed between two crossed polarizers.

The phase behavior and transition temperatures of the LC $14-2 \mathrm{M}-\mathrm{CH}_{3}$ and its nanocomposite were determined using the POM while slowly cooling from the isotropic liquid $\left(0.5^{\circ} \mathrm{C} / \mathrm{min}\right)[25,38]$. The transition temperatures of the pristine bent-core $\mathrm{LC} 14-2 \mathrm{M}-\mathrm{CH}_{3}$ were also determined previously using differential scanning calorimetry at a scan rate of $5^{\circ} \mathrm{C} / \mathrm{min}$ (reported elsewhere) [36]. The transition temperatures of the pristine $\mathrm{LC}$ and its nanocomposite as obtained from the POM observations are summarized in Table I. The dielectric measurements were carried out in the frequency range of $20 \mathrm{~Hz}-2 \mathrm{MHz}$ using an Agilent E4980A precision LCR meter. The measuring voltage was kept at $V_{\mathrm{rms}}=0.2 \mathrm{~V}$. For transmission-dependent birefringence measurements and the related order parameter calculations, the sample was placed between two crossed Glan-Thompson polarizers (GTH10M, Thorlabs, Inc.) and perpendicularly illuminated with a He-Ne laser $(\sim 633 \mathrm{~nm})[39,40]$. The rubbing direction $\vec{r}$ (i.e., the LC director $\widehat{n})$ of the planar LC cell was kept at $45^{\circ}$ with respect to the polarizer $(P)$ /analyzer $(A)$ pass axes. Transmitted power at the output end was measured using a Gentec PH100-Si-HAOD1 photodetector attached to a Gentec Maestro power meter.

\section{RESULT AND DISCUSSION}

\section{A. Polarizing optical microscopy}

The LC material is introduced in a 5- $\mu \mathrm{m}$ planar LC cell via capillary action around $10^{\circ} \mathrm{C}$ above the isotropic-nematic

TABLE I. Phase sequence and transition temperatures observed in this paper (using POM) during slow cooling.

\begin{tabular}{lc}
\hline \hline Compound & $\begin{array}{c}\text { Phase sequence and transition } \\
\text { temperatures }\left({ }^{\circ} \mathrm{C}\right)\end{array}$ \\
\hline $14-2 \mathrm{M}-\mathrm{CH}_{3}$ & Iso $134 N_{\mathrm{Cyb}} 106$ Cryst. \\
$14-2 \mathrm{M}-\mathrm{CH}_{3}+0.5 \mathrm{wt} \%$ QDs & Iso $134 N_{\mathrm{Cyb}} 104$ Cryst. \\
\hline \hline
\end{tabular}

(Iso- $N$ ) transition temperature, and the textures were recorded between crossed polarizers. The textures recorded for the LC 14-2M- $\mathrm{CH}_{3}$ and its $0.5 \mathrm{wt} \%$ QDs dispersed nanocomposite, during slow cooling from the isotropic liquid, are shown in Fig. 2. The textures of the LC nanocomposite exhibited fairly homogeneous colors (and, hence, alignment) similar to that of the pristine LC. This indicates a good homogeneous dispersion of QDs in the LC matrix without any aggregation [25]. Close to the Iso- $N$ transition temperature, we observe a sharp color change owing to the development of nematic order in these systems [see Figs. 2(a) and 2(f)]. As the temperature is further lowered, uniform marble textures, typical of the nematic phase, appear with colors varying with temperature [36]. The isotropic-nematic transition temperature remains nearly unaltered after the incorporation of QDs. In the $N$ phase, the emergent colors change with decreasing temperature, which indicates that the birefringence $(\Delta n)$ also changes with temperature. A qualitative measurement of this change in birefringence can be performed by matching the colors with the Michel-Levy chart for a given thickness [41]. We deduce that $\Delta n$ increases with decreasing temperature from this mapping. Also, the change in $\Delta n$ with temperature is found to be quite high $(\sim 0.06)$. This is suggestive of highly ordered microstructures in the $N$ phase of the BLC compound $[6,42]$. Also, from Fig. 2 we can clearly see that the temperature-dependent textural color sequence changes or shifts after incorporation of the QDs. With the help of the Michel-Levy chart, we qualitatively deduce that the $\Delta n$ values, for a fixed temperature, are lowered on the incorporation of the QDs, implying a reduction in the corresponding nematic order parameter $S$ since $\Delta n \propto S$ [38]. Experimentally, $\Delta n$ measurements and the associated order parameter $(S)$ calculations have also been performed, and they are discussed in detail in the Sec. III B.

\section{B. Optical birefringence measurements and orientational order parameter calculations}

The birefringence $(\Delta n)$ measurements of the LC sample and its nanocomposite as a function of temperature have been performed with the optical transmission technique. The planar LC sample is perpendicularly illuminated with a $\mathrm{He}-\mathrm{Ne}$ laser $(\lambda \sim 633 \mathrm{~nm})$ and placed between two crossed GlanThompson polarizers such that the optic axis makes an angle $\varphi=45^{\circ}$ with the polarizer or analyzer pass axis. The power at the output end is measured with a photodetector. The transmitted light intensity is then given in terms of the phase retardation $(\delta)$ as [43]

$$
I=I_{0} \sin ^{2} 2 \varphi \sin ^{2} \frac{\delta}{2}=\frac{I_{0}}{2}(1-\cos \delta) .
$$

Here, $\delta=\frac{2 \pi}{\lambda} \Delta n d$ is the phase retardation, $I$ is the transmitted light intensity, $I_{0}$ is the incident light intensity, $\varphi$ (= $45^{\circ}$ ) is the azimuthal angle, i.e., the angle made by optic axis with the polarizer or analyzer pass axis, $\lambda$ is the incident light wavelength, $\Delta n=n_{e}-n_{o}$ is the birefringence, $n_{e}$ and $n_{o}$ are the extraordinary and ordinary refractive indices of the LC, respectively, and $d$ is the thickness of the LC cell. The birefringence $\Delta n$ is measured directly from the experimental results using Eq. (1) as a function of temperature. In Fig. 3, we 

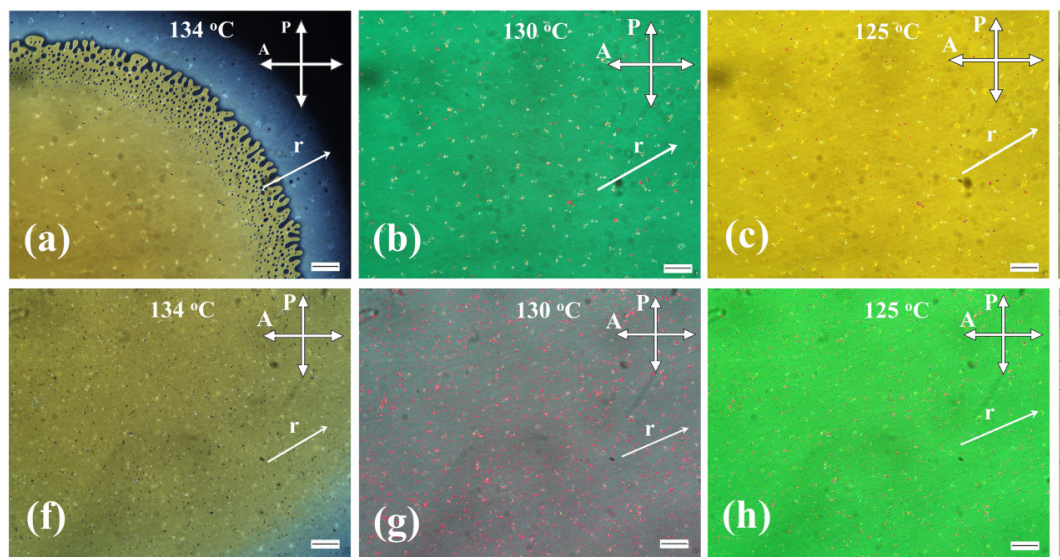
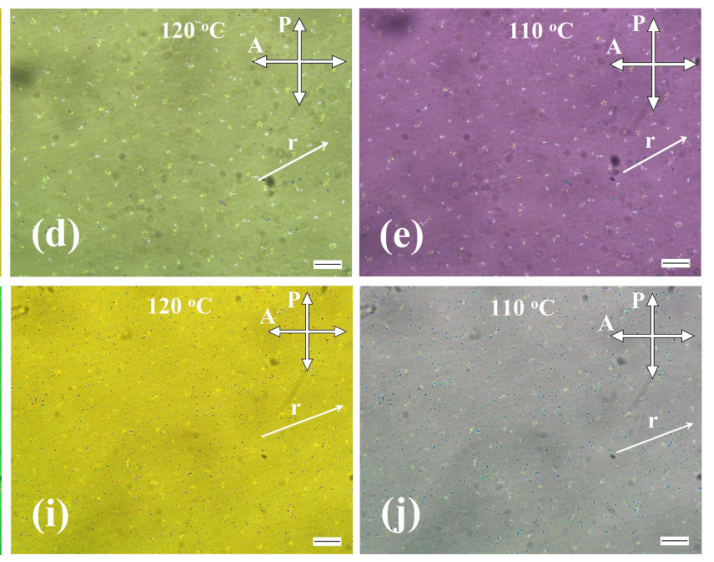

FIG. 2. Birefringent textural color variation with temperature of (a)-(e) the bent-core $\mathrm{LC} 14-2 \mathrm{M}-\mathrm{CH}_{3}$ and (f)-(j) the $0.5 \mathrm{wt} \% \mathrm{CdSe} / \mathrm{ZnS}$ QDs dispersed 14-2M- $\mathrm{CH}_{3}$, respectively, during cooling. In each image, $\boldsymbol{r}$ indicates the rubbing direction, and the scale bar indicates $100 \mu \mathrm{m}$. The periodic white spots in the background of the images are features of the LC cell (Instec Inc., USA) caused by the PI printing fabric in the production line.

plot the experimentally measured birefringence $(\Delta n)$ values for pure $14-2 \mathrm{M}-\mathrm{CH}_{3}$ (half-filled squares) and its nanocomposite (half-filled circles) at different temperatures. For both cases, on cooling from the isotropic liquid, $\Delta n$ manifests a sharp increase following the isotropic- $N$ phase transition, basically due to an enhancement in the nematic order. On further cooling, $\Delta n$ retains the trend, but now the increase is relatively slow. It is to be noted that the birefringence values decrease appreciably due to the incorporation of QDs in the entire mesophase range.

For precise determination of the temperature dependence of the nematic order parameter $(S)$, we resort to the fourparameter power-law expression, which is in agreement with

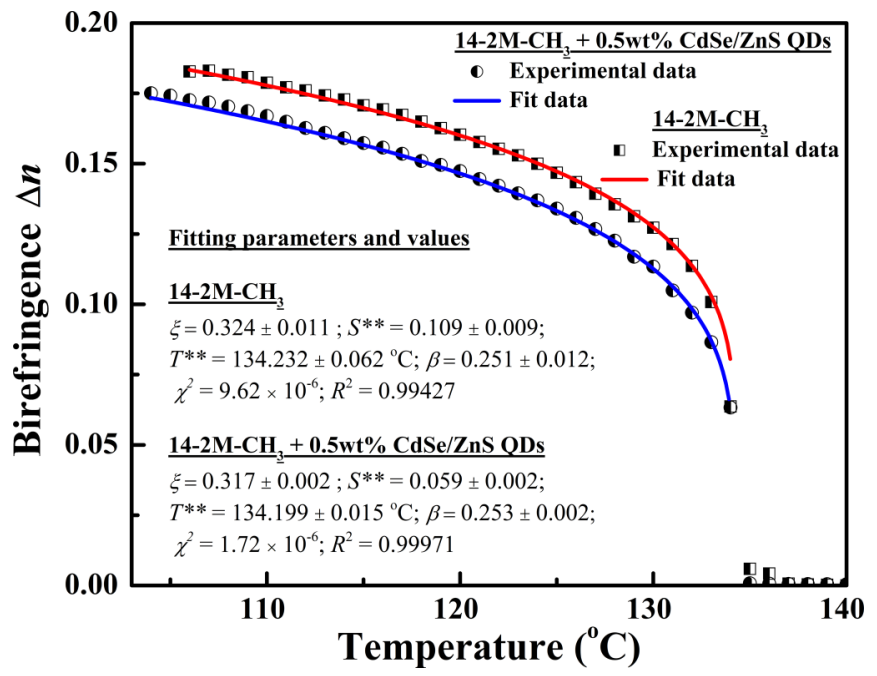

FIG. 3. Experimental values of birefringence $(\Delta n)$ for the LC $14-2 \mathrm{M}-\mathrm{CH}_{3}$ (half-filled squares) and its nanocomposite (half-filled circles); the solid lines (pure LC: red and LC nanocomposite: blue) represent the four-parameter fit to the experimental data using Eq. (3). The related fitting parameter values are shown in the figure. The fitting parameters $\chi^{2}$ and $R^{2}$ are generated by the fitting algorithm so that $\chi^{2} \sim 0$ and $R^{2} \sim 1$ describe good fits. the mean-field theory of weakly first-order transitions $[39,44]$,

$$
S(T)=S^{* *}+A\left|\left(1-\frac{T}{T^{* *}}\right)\right|^{\beta} .
$$

Here, $T$ is the absolute temperature, $T^{* *}$ is the absolute superheating limit of the nematic phase; at $T=T^{* *}, S\left(T^{* *}\right)=$ $S^{* *}, \beta$ is the critical exponent, and $A$ is a constant. At $T=$ $0, S(0)=1$, which implies $1=S^{* *}+A$. The birefringence $(\Delta n)$ can then be expressed as [39]

$$
\Delta n=\xi\left[S^{* *}+\left(1-S^{* *}\right)\left|\left(1-\frac{T}{T^{* *}}\right)\right|^{\beta}\right],
$$

where, $\xi=(\Delta \alpha /\langle\alpha\rangle)\left[\left(n_{I}^{2}-1\right) / 2 n_{I}\right], \Delta \alpha$ is the molecular polarizability anisotropy, $\langle\alpha\rangle$ is the mean polarizability, and $n_{I}$ is the refractive index in the isotropic phase just above the isotropic- $N$ transition temperature. The experimental birefringence $(\Delta n)$ data has been well fitted with Eq. (3), which involves four fit parameters $\xi, S^{* *}, \beta$, and $T^{* *}$. The obtained fitting plots (pure LC: red solid line and LC nanocomposite: blue solid line) along with the fit parameter values are shown in Fig. 3. The four-parameter fitting is considered superior to the Haller's method that involves lesser number of fit parameters $[39,44,45]$. We obtain $\xi=0.324, S^{* *}=0.109, T^{* *}=$ $134.232^{\circ} \mathrm{C}$, and $\beta=0.251$ for the pure LC. For the LC nanocomposite, we obtain $\xi=0.317, S^{* *}=0.059, T^{* *}=$ $134.199^{\circ} \mathrm{C}$, and $\beta=0.253$. The fit parameter values remain almost unaltered after the incorporation of QDs except the value of $S^{* *}$, which is reduced almost by a factor of $\frac{1}{2}$. It is indicative that the QDs have a significant effect on the nematic order in the LC mesophase. The value of the critical exponent $\beta$ is around 0.25 in both cases, which is in excellent agreement with the theoretically predicted values for the nematic phase $[39,44]$. The temperature-dependent macroscopic orientational order parameter $(S)$ is calculated using Eq. (2) and using the parameter values obtained from the fittings. The obtained temperature-dependent profiles of $S$ for both cases are shown in Fig. 4. The order parameter $S$ decreases appreciably after the incorporation of QDs. The decrease in order parameter can be ascribed to the reduction of cybotactic 


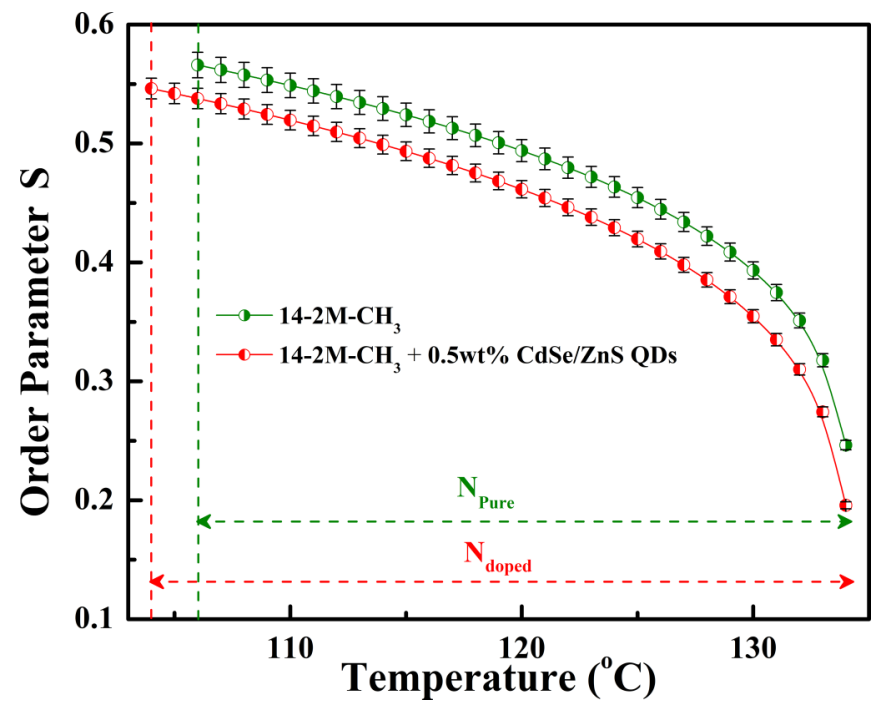

FIG. 4. Orientational order parameter $(S)$ as a function of temperature for the bent-core $\mathrm{LC} 14-2 \mathrm{M}-\mathrm{CH}_{3}$ and its $0.5 \mathrm{wt} \%$ $\mathrm{CdSe} / \mathrm{ZnS}$ QDs incorporated nanocomposite.

cluster size after QDs incorporation as will be discussed in the dielectric studies section. The nematic phase range as observed from the birefringence measurements were found around $134-106{ }^{\circ} \mathrm{C}$ for the pure LC and around $134-104^{\circ} \mathrm{C}$ for the QDs incorporated LC, complying with the POM observations.

\section{Dielectric studies}

Dielectric measurements have been carried out in a frequency range of $20 \mathrm{~Hz}-2 \mathrm{MHz}$ (measuring voltage $V_{\mathrm{rms}}=$ $0.2 \mathrm{~V}$ ) and at different temperatures during the cooling cycle. The complex dielectric permittivity $\left(\epsilon^{*}\right)$ of LCs, in the frequency domain, is expressed as $\epsilon^{*}(f)=\epsilon^{\prime}(f)-i \epsilon^{\prime \prime}(f)$ [46]. Here, $\epsilon^{\prime}$ and $\epsilon^{\prime \prime}$ are the real and the imaginary parts of the complex dielectric permittivity, respectively. The dielectric spectra of $\epsilon^{\prime}$ and $\epsilon^{\prime \prime}$, obtained from experiments, for the LC 14-2M-CH 3 and its QD-dispersed nanocomposite are shown in Fig. 5. The maximum experimental error for the dielectric measurements lie within $\pm 1 \%$. From Fig. 5(a), we can see that the value of $\epsilon^{\prime}$ at lower frequencies is $\sim 110$ for the $\mathrm{LC} 14-2 \mathrm{M}-\mathrm{CH}_{3}$. Such high values of $\epsilon^{\prime}$ have been recently observed in bent-core LCs containing cybotactic clusters [10,11]. The dielectric absorption spectra unveils the associated relaxation processes in the medium. The absorption spectra of $14-2 \mathrm{M}-\mathrm{CH}_{3}$ is depicted in Fig. 5(b). At any temperature, two distinct relaxation peaks (or modes) can be identified: a low-frequency mode $\left(M_{1}\right)$ and a highfrequency mode $\left(M_{2}\right)$. The two modes represent different relaxation processes present in the system. Collective relaxation processes (due to cybotactic clusters) are known to give rise to low-frequency absorption peaks similar to $M_{1}$, and they are widely encountered in the $N$ phases of bent-core LCs $[10-12,14,46,47]$. The relaxation frequencies $\left(f_{R}\right)$ associated with cybotactic clusters can vary in the range of few tens of hertz to a few hundred hertz [10-12,14]. Therefore, mode $M_{1}$ is attributed to collective relaxation processes originating
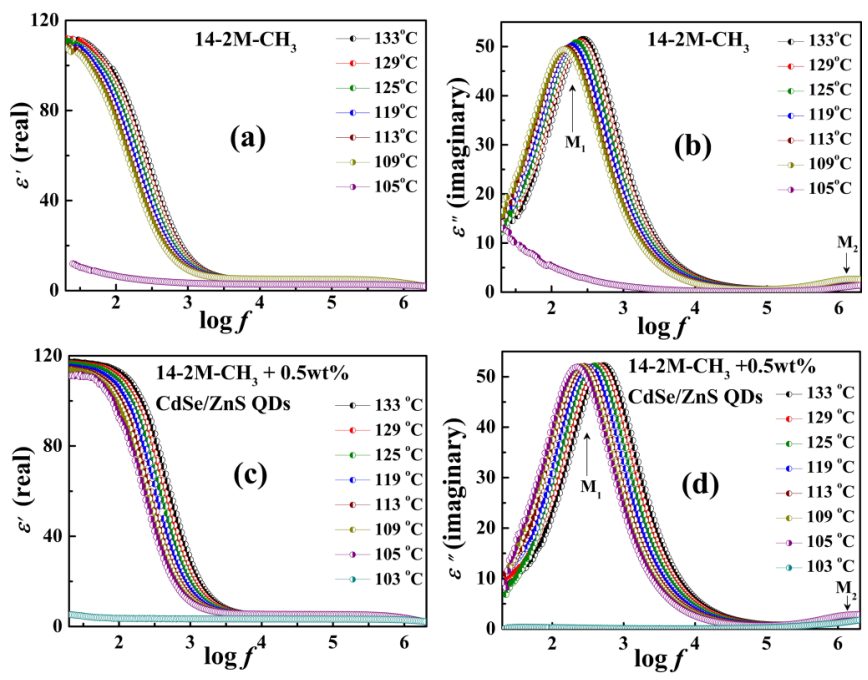

FIG. 5. Frequency-dependent real $\left(\epsilon^{\prime}\right)$ and imaginary $\left(\epsilon^{\prime \prime}\right)$ parts of dielectric permittivity of (a) and (b) pristine LC (14-2M- $\left.\mathrm{CH}_{3}\right)$ and (c) and (d) QDs dispersed LC nanocomposite (14-2M- $\mathrm{CH}_{3}+$ 0.5 wt $\% \mathrm{CdSe} / \mathrm{ZnS}$ QDs) at different temperatures during cooling ( $f$ in hertz).

from cybotactic clusters present in the $N$ phase of the LC. These clusters only occupy a fraction of the volume, and not all molecules form these clusters [34,35]. Experiments show that the clusters can also exist in the isotropic phase, and their size does not change significantly across the Iso- $N$ transition-a unique property of BLCs that warrants further investigation [12,35,36,48,49]. As reported in Ref. [36], through detailed small-angle x-ray scattering and dielectric measurements, the $N$ phase of the pristine LC $14-2 \mathrm{M}-\mathrm{CH}_{3}$ is cybotactic in nature, i.e., it contains smecticlike cybotactic clusters. Also, mode $M_{1}$ is not associated with ionic impurities because no polarization response (ionic) could be detected for both the pure and the doped LCs (applied voltage up to $80 V_{p p}$, frequencies between the millihertz to the kilohertz range) $[3,36]$. The high-frequency mode $M_{2}$ may have resulted due to the cell's ITO electrodes. In a cell filled with LCs, the characteristic frequency of the ITO relaxation may decrease, and it may become temperature dependent due to finite impedance- and temperature-dependent dielectric constants of the LC. Mode $M_{2}$ manifested decreasing dielectric strength with increasing temperature, and it was absent in the crystalline phase. In a few studies reported in the literature, high-frequency modes, similar to $M_{2}$, were identified as the molecular processes (e.g., reorientation of the short or long axis) $[12,36,50]$. Therefore, we deduce that $M_{2}$ may have resulted from the cell's ITO electrodes or from a combination of the cell's ITO and the molecular processes.

For the 0.5 wt $\%$ QDs incorporated $14-2 \mathrm{M}-\mathrm{CH}_{3}$, the dispersion curve is shown in Fig. 5(c). Similar to the pristine LC 14-2M- $-\mathrm{CH}_{3}$, we can see that the value of $\epsilon^{\prime}$ at lower frequencies is large $(\sim 110)$. The absorption spectra of the LC nanocomposite is depicted in Fig. 5(d). At any temperature, two distinct relaxation peaks (or modes) can be identified: a low-frequency mode $\left(M_{1}\right)$ and a high-frequency mode $\left(M_{2}\right)$. After the incorporation of QDs, a relative change in the associated relaxation frequencies $\left(f_{R}\right)$ of the modes can be 

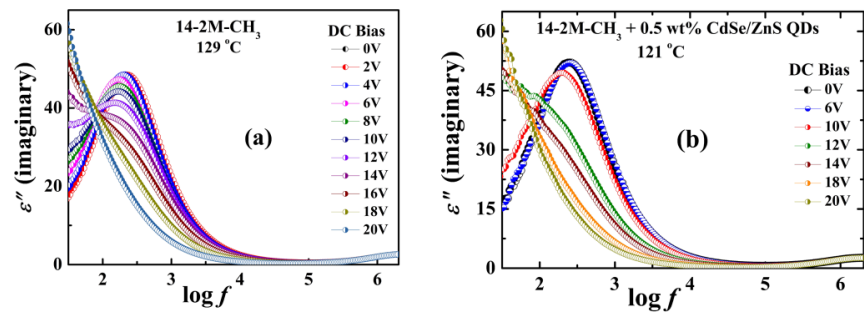

FIG. 6. DC bias suppression of the low-frequency relaxation mode $\left(M_{1}\right)$ in (a) pure $14-2 \mathrm{M}-\mathrm{CH}_{3}$ at $129^{\circ} \mathrm{C}$ and (b) $0.5 \mathrm{wt} \%$ QDs incorporated $14-2 \mathrm{M}-\mathrm{CH}_{3}$ at $121^{\circ} \mathrm{C}(f$ in hertz).

observed, compared to the pristine LC. The values of $f_{R}$ have been evaluated from the experimental data, and it has been discussed in detail later in this section. The $f_{R}$ associated with $M_{1}$ is denoted by $f_{R 1}$ and for $M_{2}$, it is denoted by $f_{R 2}$. By comparison with the results obtained for the pristine LC $14-2 \mathrm{M}-\mathrm{CH}_{3}$, it is evident that the collective processes (and, hence, the cybotactic clusters) survive in the QD-dispersed LC nanocomposite. However, to establish this firmly, additional DC bias measurements have been performed. For collective processes, when a DC bias voltage is applied across a LC cell, the relaxation process ceases to exist. As a result, the dielectric relaxation modes get suppressed, and at high voltages, they become extinct $[12,46,51]$. A DC bias voltage of amplitude up to $20 \mathrm{~V}$ was applied across the LC cell, and the dielectric measurements were performed (Fig. 6). For the pure LC $14-2 \mathrm{M}-\mathrm{CH}_{3}$, a continuous and gradual suppression of mode $M_{1}$ with an applied DC bias voltage is observed [Fig. 6(a)]. It is a confirmatory proof of collective relaxations and, hence, the presence of cybotactic clusters in the $N$ phase of the LC $[12,36]$. Similar to the pristine LC, we observe that the mode $M_{1}$ of the LC nanocomposite becomes suppressed [Fig. 6(b)], and then completely absent at higher voltages $(\sim 20 \mathrm{~V})$. This observation further confirms the collective behavior of $M_{1}[12,36]$ and, hence, corroborates retention of the cybotactic nematic phase $\left(N_{\mathrm{Cyb}}\right)$ in the QD-dispersed LC nanocomposite. The high-frequency mode $M_{2}$, however, does not show any change after DC bias application. Moreover, we note that in the doped LC, similar to the pristine $\mathrm{LC}, M_{2}$ is absent in the crystalline state, and its strength decreases with increasing temperature.

The permittivity $\left(\epsilon^{\prime}\right)$ values at $f=10 \mathrm{kHz}$ have been evaluated as a function of temperature (Fig. 7). It shows that on incorporation of QDs, the permittivity $\left(\epsilon^{\prime}\right)$ increases appreciably. In a planar configuration, $\epsilon^{\prime}$ represents $\epsilon_{\perp}$. The dielectric anisotropy $(\Delta \epsilon)$ is defined as $\Delta \epsilon=\epsilon_{\|}-\epsilon_{\perp}$. Therefore, an increase in $\epsilon^{\prime}$ implies a decrease in $\Delta \epsilon$. Furthermore, a reduction in the dielectric anisotropy is indicative of decreasing macroscopic order parameter (since $\Delta \epsilon \propto S$ ) [25,33]. It agrees well with the observations made in Secs. III B and III A.

To analyze the dielectric modes and the effects of incorporation of QDs, the associated dielectric parameters [e.g., dielectric strength $(\delta \epsilon)$, relaxation frequency $\left(f_{R}\right)$ ] have been evaluated by fitting the experimental dielectric data (both $\epsilon^{\prime}$ and $\epsilon^{\prime \prime}$, simultaneously), using the well-known HavriliakNegami (H-N) fit function. The frequency-dependent complex dielectric permittivity $\epsilon^{*}(f)$ can be described by the modified H-N equation [40,52-55], which also includes contributions

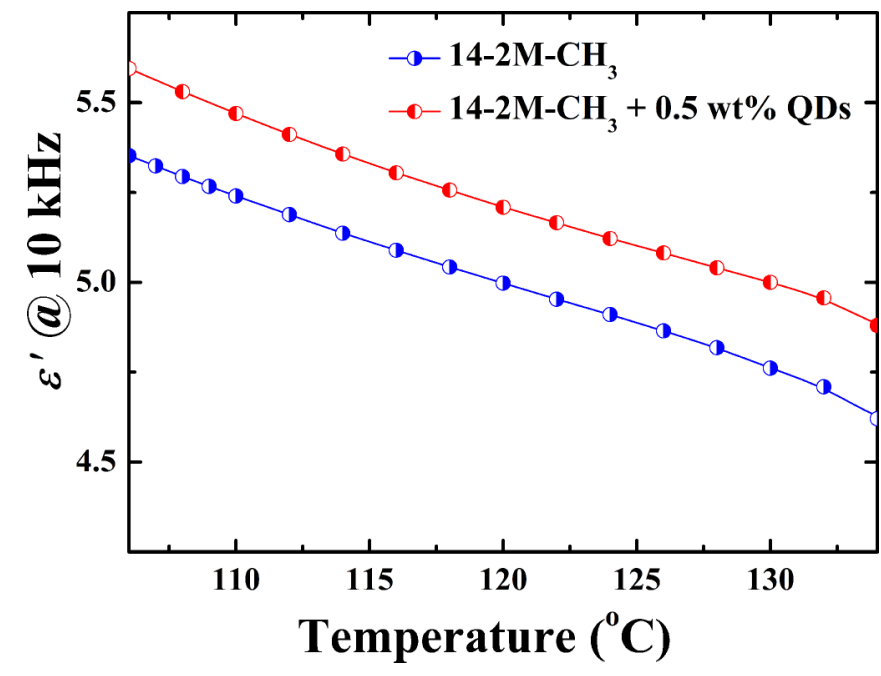

FIG. 7. Dielectric permittivity of $14-2 \mathrm{M}-\mathrm{CH}_{3}$ and its nanocomposite at $10 \mathrm{kHz}$ as a function of temperature.

from the DC conductivity $\left(\sigma_{0}\right)$,

$$
\epsilon^{*}(f)=\epsilon_{\infty}+\sum_{k=1}^{N} \frac{\delta \epsilon_{k}}{\left[1+\left(i 2 \pi f \tau_{k}\right)^{\alpha_{k}}\right]^{\beta_{k}}}-\frac{i \sigma_{0}}{\epsilon_{0}(2 \pi f)^{s}} .
$$

The last term on the right-hand side of Eq. (4) describes the motion of free-charge carriers in the sample. The characteristic dielectric parameters, such as the relaxation frequency $\left(f_{R}\right)$ and the dielectric strength $(\delta \epsilon)$ are obtained by fitting the experimental dielectric permittivity $\left(\epsilon^{\prime}\right)$ and dielectric loss $\left(\epsilon^{\prime \prime}\right)$ data simultaneously to the real and the imaginary parts of Eq. (4) given by $[12,40,54-56]$

$$
\begin{aligned}
\epsilon^{\prime}= & \epsilon_{\infty} \\
& +\sum_{k=1}^{N} \frac{\delta \epsilon_{k} \cos \left(\beta_{k} \theta\right)}{\left[1+\left(2 \pi f \tau_{k}\right)^{2 \alpha_{k}}+2\left(2 \pi f \tau_{k}\right)^{\alpha_{k}} \cos \left(\alpha_{k} \pi / 2\right)\right]^{\beta_{k} / 2}}, \\
\epsilon^{\prime \prime}= & \frac{\sigma_{0}}{\epsilon_{0}(2 \pi f)^{s}} \\
& +\sum_{k=1}^{N} \frac{\delta \epsilon_{k} \sin \left(\beta_{k} \theta\right)}{\left[1+\left(2 \pi f \tau_{k}\right)^{2 \alpha_{k}}+2\left(2 \pi f \tau_{k}\right)^{\alpha_{k}} \cos \left(\alpha_{k} \pi / 2\right)\right]^{\beta_{k} / 2}} .
\end{aligned}
$$

Here,

$$
\theta=\tan ^{-1}\left[\frac{\left(2 \pi f \tau_{k}\right)^{\alpha_{k}} \sin \left(\alpha_{k} \pi / 2\right)}{1+\left(2 \pi f \tau_{k}\right)^{\alpha_{k}} \cos \left(\alpha_{k} \pi / 2\right)}\right]
$$

Here, $f$ is the frequency, $\epsilon_{\infty}$ is the high-frequency limit of permittivity, $\delta \epsilon_{k}$ is the dielectric strength for the $k$ th relaxation process, $\sigma_{0}$ is the DC conductivity, $\epsilon_{0}$ is the free-space permittivity $\left(8.854 * 10^{-12} \mathrm{~F} / \mathrm{m}\right), s$ is a fitting parameter responsible for the nonlinearity in the DC conductivity part (for ohmic behavior, $s=1$ ), $k$ is the number of relaxation processes, $\tau_{k}\left(=1 / 2 \pi f_{k}\right)$ is the relaxation time for the $k$ th relaxation process, $\alpha_{k}$ and $\beta_{k}$ are the empirical fit parameters that describe symmetric and nonsymmetric broadening, 

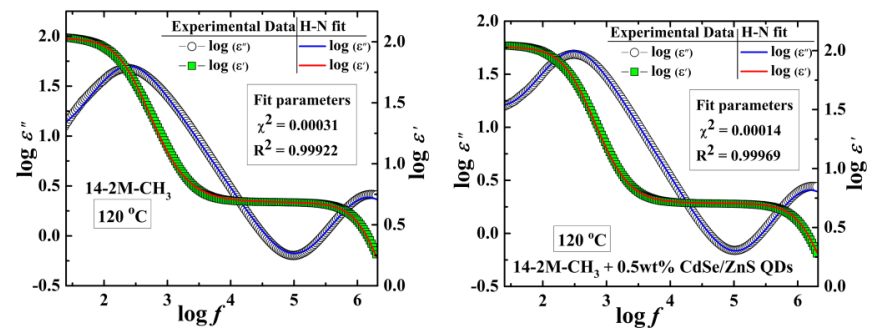

FIG. 8. Simultaneous fitting of the real $\left(\epsilon^{\prime}\right)$ and the imaginary $\left(\epsilon^{\prime \prime}\right)$ parts of complex dielectric permittivity (on the logarithmic scale) using the $\mathrm{H}-\mathrm{N}$ equations in (a) pure $14-2 \mathrm{M}-\mathrm{CH}_{3}$ and (b) 0.5 wt $\%$ QDs incorporated $14-2 \mathrm{M}-\mathrm{CH}_{3}$ ( $f$ in hertz). Experimental data-The green squares represent $\epsilon^{\prime}$, and the hollow circles represent $\epsilon^{\prime \prime}$. Fit data-The red solid line represents fit to $\epsilon^{\prime}$, and the blue solid line represents fit to $\epsilon^{\prime \prime}$. The fitting parameters $\chi^{2}$ and $R^{2}$ are generated by the fitting algorithm so that $\chi^{2} \sim 0$ and $R^{2} \sim 1$ describe good fits.

respectively, of the $k$ th relaxation peak. In our case, in the absorption curve, we have two different relaxation peaks and, hence, $k=1$ and 2 . A representative of the obtained H-N fits are shown in Fig. 8. The values of $\alpha_{1}$ and $\alpha_{2}$ lie in the range of $0.97-1$ whereas the values of $\beta_{1}$ and $\beta_{2}$ lie in the range of 0.93-1 (we perform the fitting over a range of temperatures $106^{\circ} \mathrm{C}-134{ }^{\circ} \mathrm{C}$ and the ranges in $\alpha_{1} \cdots \beta_{2}$ are specified). In the study of a five-ring bent-core LC C1Pbis10BB and its mixtures with a calamitic nematic LC 6OO8, the authors reported a Debye-type low-frequency relaxation mode $B_{\| 1}$ [57]. They also write that smecticlike clusters can induce a Debye-type relaxation in the low-frequency region of dielectric spectrum. Our dielectric results also indicate that $M_{1}$ is a near Debye-like relaxation process and the associated relaxation frequencies overlap with the mode $B_{\| 1}$ reported in Ref. [57]. The variations of the relaxation frequency $\left(f_{R}\right)$ and the dielectric strength $(\delta \epsilon)$ of modes $M_{1}$ and $M_{2}$ with temperature as obtained from the fitting are shown in Fig. 9. The results show that $\delta \epsilon_{1}$ (i.e., corresponding to $M_{1}$ ) for $14-2 \mathrm{M}-\mathrm{CH}_{3}$ increases slightly from $\sim 98$ to $\sim 104$ with increasing temperature. Similarly, $\delta \epsilon_{1}$ for the QDs dispersed nanocomposite increases slightly, from $\sim 100$ to $\sim 105$ with increasing temperature. Thus, the dielectric strength $\delta \epsilon_{1}$ is largely unaffected on doping. Again, the value of $\delta \epsilon_{1}$ is quite large, and it is similar to other bent-core LCs with cybotactic clusters [10-12]. The dielectric strength $\delta \epsilon_{2}$ associated with $M_{2}$ is found to be very small and increases with decreasing temperature from $\sim 4.5$ to 5.5 for both $14-2 \mathrm{M}-\mathrm{CH}_{3}$ and its nanocomposite.

The relaxation frequency $\left(f_{R 1}\right)$ associated with $M_{1}$ lies in the range of $\sim 170$ to $\sim 320 \mathrm{~Hz}$ for the pure LC, similar to several other bent-core LCs with cybotactic clusters [10-12]. The incorporation of QDs causes $f_{R 1}$ to shift to higher frequencies $(\sim 220$ to $\sim 430 \mathrm{~Hz})$. It indicates that there is an apparent reduction in size of the smecticlike cybotactic clusters [48]. This reduction can be estimated qualitatively by taking the ratio of the relaxation frequency $f_{R 1}$ for the pristine LC and its doped counterpart. The ratio has an average value of $\sim 0.67$ where the average is taken over two sets of measurements and a range of temperatures. This ratio signifies a relative change in the average number of molecules $\left(N_{c}\right)$ present in each cluster (in accordance with our earlier theoretical model and the
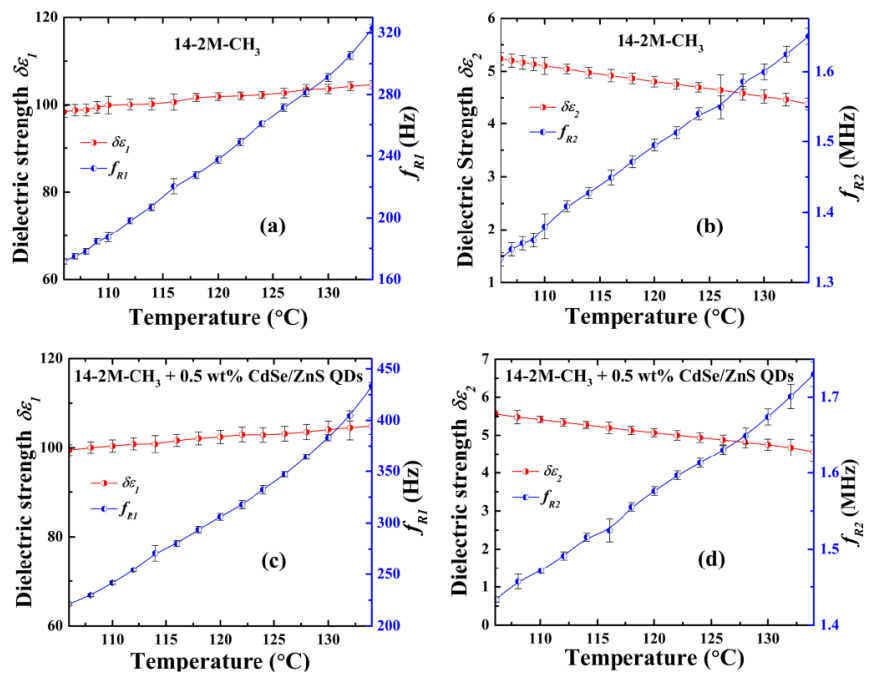

FIG. 9. Temperature-dependent variation of the relaxation frequency $\left(f_{R}\right)$ and the dielectric strength $(\delta \epsilon)$ corresponding to $M_{1}$ and $M_{2}$ of (a) and (b) the pristine LC and (c) and (d) $0.5 \mathrm{wt} \%$ QDs incorporated LC.

experiments) $[34,48]$. The decrease in the measured order parameter $(S)$ on doping can be ascribed to reduced cluster sizes on QDs incorporation. In our earlier theoretical work on bentcore nematic LCs, we take $N_{c}=50$ [34]. Recent observations have shown that the typical size of smecticlike cybotactic clusters lie in the range of a few tens of nanometers to around a hundred nanometers [47]. Again, the typical dimension of a bent-core LC molecule is around 2 to $3 \mathrm{~nm}$. Therefore, the number $N_{c}=50$ is justified in the case of pure (undoped) bent-core LCs, with cybotactic clusters. For the QD-dispersed bent-core nematic LCs, we can take $N_{c} \sim 33(=50 \times 0.67)$ as a reasonable value. The relaxation frequency $f_{R 1}$ manifests a gradual decrease with decreasing temperature, divulging an Arrhenius-type behavior $\left[f_{R}=f_{0} \exp \left(-E_{a} / k_{B} T\right) ; f_{0}\right.$ is a temperature-independent constant, $E_{a}$ is the activation energy, $k_{B}$ is the Boltzmann constant, and $T$ is the absolute temperature]. $f_{R 2}$ also demonstrates an Arrhenius-like behavior.

The activation energy $\left(E_{a}\right)$ associated with a relaxation process represents the minimum amount of energy required for that process to take place [46]. The value of $E_{a}$ associated with the relaxation processes can be obtained by plotting $f_{R}$ as a function of $1 / T$, using the relation $f_{R}=$ $f_{0} \exp \left(-E_{a} / k_{B} T\right)$. The Arrhenius plots of $\ln \left(f_{R 1}\right)$ vs $1 / T$ (for $M_{1}$ ) for the two compounds are shown in Fig. 10. The activation energy $\left(E_{a}\right)$ associated with $M_{1}$ is evaluated from the slope of the linear fit as shown in Fig. 10. The value of $E_{a}(\sim 29.12 \mathrm{~kJ} / \mathrm{mol})$ increases significantly after the incorporation of QDs $(\sim 37.68 \mathrm{~kJ} / \mathrm{mol})$. For a small cluster size, the cluster's dipole moment $(\mu)$ also becomes small. Hence, more energy is required to interact with an external electric field. Therefore, an increased value of $E_{a}$ for $M_{1}$, after the incorporation of $\mathrm{CdSe} / \mathrm{ZnS}$ QDs, implies a decrease in the size of cybotactic clusters. It also concurs with our earlier observations. The activation energy associated with $M_{2}$ has rather small values $(\sim 8 \mathrm{~kJ} / \mathrm{mol})$, and it does not change significantly after the incorporation of QDs. 

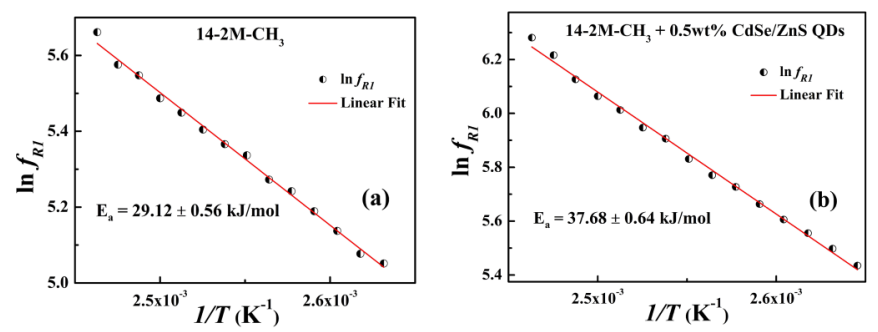

FIG. 10. Arrhenius plot of the $M_{1}$ relaxation frequency $\left(f_{R 1}\right)$ in the $N$ phase of (a) pristine and (b) 0.5 -wt $\% \mathrm{CdSe} / \mathrm{ZnS}$ QDs incorporated $14-2 \mathrm{M}-\mathrm{CH}_{3}$. The activation energy $\left(E_{a}\right)$ is calculated from the slope of the linear fit, represented by the solid red line.

\section{MATHEMATICAL MODEL}

In this section, we propose a simple mathematical model that describes the QD doping-induced reduction of the nematic scalar order parameter for dilute suspensions that could, subsequently, be improved to describe novel features, such as ferroelectricity, chirality, biaxiality, and transition pathways between multiple stable states. Since the experimental domain is a simple planar cell, denoted by $\Omega \subset \mathbb{R}^{3}$, with a height about $5 \mu \mathrm{m}\left(5 \times 10^{-6} \mathrm{~m}\right)$, we assume a characteristic length of the system,

$$
x_{s}=5 \times 10^{-8} \mathrm{~m}
$$

as in Ref. [34] so that the cell thickness is 100 units of $x_{s}$. The cross-sectional dimensions of the cell are much larger than the cell height, so it is reasonable to assume that structural variations only occur across the cell height, i.e., this is a onedimensional problem. We assume that the QDs are spherical in shape with an average radius of $2.8 \mathrm{~nm}$; the size of the QDs is much smaller than the typical separation between them, and the total volume occupied by the QDs is small. Let $R$ denote the radius of a QD (2.8 $\mathrm{nm}$ as reported in these experiments), and we define a small parameter $\epsilon$ so that,

$$
\epsilon^{\alpha}=\frac{R}{x_{s}}=R_{0}=0.056
$$

for some $1<\alpha<\frac{3}{2}$. The definition of $\epsilon$ is not unique, provided it is a small parameter, relevant for dilute uniform suspensions of QDs [37]. In particular, our mathematical model is restricted to dilute suspensions and will need to be modified for nondilute systems.

In Ref. [35], Madhusudana proposes a LdG-type two-state model for the $N$ phase of BLCs, accounting for cybotactic clusters. This two-state model is a phenomenological model based on the premise that the $N$ phase of the BLC comprises two different types of molecules: ground state (GS) molecules and excited state (ES) molecules. The ES molecules define the smecticlike cybotactic clusters, and the GS molecules are located outside the clusters. The generic LdG theory models a single component system, e.g., the GS molecules, typically assumed to be rodlike molecules that tend to align with each other, yielding long-range orientational order [58]. Madhusudana's model is a two component model, the GS and ES molecules with additional coupling effects. In Ref. [34], we describe the $N$ phase of the BLC by two macroscopic tensor order parameters (with a number of simplifying assumptions),

$$
\begin{aligned}
& \mathbf{Q}_{g}=\sqrt{\frac{3}{2}} S_{g}\left(\mathbf{n}_{g} \otimes \mathbf{n}_{g}-\frac{1}{3} I\right), \\
& \mathbf{Q}_{c}=\sqrt{\frac{3}{2}} S_{c}\left(\mathbf{n}_{c} \otimes \mathbf{n}_{c}-\frac{1}{3} I\right),
\end{aligned}
$$

respectively, where $\mathbf{Q}_{g}$ is the $\mathrm{LdG}$ order parameter for the GS molecules and $\mathbf{Q}_{c}$ is the $\mathrm{LdG}$ order parameter associated with the ES molecules. In both cases, we assume that $\mathbf{Q}_{g}$ and $\mathbf{Q}_{c}$ have uniaxial symmetry, i.e., the GS (ES) molecules align along a single averaged distinguished direction $\mathbf{n}_{g}$ (respectively, $\mathbf{n}_{c}$ ) and assume that $\mathbf{n}_{g}$ and $\mathbf{n}_{c}$ are constant unit vectors or directions so that there are no director distortions or defects. There are two scalar order parameters $S_{c}$ and $S_{g}$ corresponding to ES (excited state) and GS (ground state) molecules, respectively. As is standard with variational approaches, the experimentally observed configurations are described by local or global minimizers of a suitably defined LdG-type energy in terms of $S_{c}, S_{g}$, and the coupling between them.

In Patranabish et al. [34], the authors theoretically study stable $\left(S_{g}, S_{c}\right)$ profiles in a simple planar cell geometry (as experimentally studied in this paper) as a function of the temperature, in terms of minimizers of the following LdG-type energy (heavily inspired by the work in Ref. [35] with additional elastic effects that account for spatial inhomogeneities):

$$
\begin{aligned}
\mathcal{F}= & \int_{\Omega}\left(1-a_{x}\right)\left(\frac{a_{g}}{2}\left(T-T^{*}\right) S_{g}^{2}-\frac{B_{g}}{3} S_{g}^{3}+\frac{C_{g}}{4} S_{g}^{4}-E_{\mathrm{el}} S_{g}\right) \\
& +\frac{a_{x}}{N_{c}}\left(-\left(1-a_{x}\right) \gamma S_{g} S_{c}+\frac{\alpha_{c}}{2} S_{c}^{2}+\frac{\beta_{c}}{4} S_{c}^{4}\right) \\
& -a_{x} J E_{\mathrm{el}} S_{c}+K_{g}\left|\nabla S_{g}\right|^{2}+K_{c}\left|\nabla S_{c}\right|^{2} d \mathbf{x} .
\end{aligned}
$$

Here, the subscripts $g$ and $c$ denote the GS and the ES molecules, respectively, and the clusters are essentially formed by the ES molecules. They work with a one-constant approximation and $K_{g}$ and $K_{c}$ are the elastic constants of the GS and ES molecules, respectively. $a_{g}, B_{g}$, and $C_{g}$ are the material-dependent parameters in the $\mathrm{LdG}$ free energy, and $T^{*}$ is the nematic supercooling temperature such that the isotropic phase of the GS phase is unstable for $T<T^{*}$. The parameter $\gamma$ is the coupling parameter between the GS molecules and the clusters [35]. The coefficients, $\alpha_{c}$ and $\beta_{c}$, are saturation parameters to ensure that the absolute value of $S_{c}<1$ for physically relevant parameter regimes, $N_{c}$ is the number of ES molecules in each cluster, and $a_{x}$ is the mole fraction of the ES molecules. $J$ accounts for the shape anisotropy of ES molecules. $E_{\mathrm{el}}$ is the electric field energy $\left(\frac{1}{2} \epsilon_{0} \Delta \epsilon E^{2}\right)$ where $\epsilon_{0}$ is the free-space permittivity, $\Delta \epsilon$ is the dielectric anisotropy, $E$ is the applied electric field.

The mathematically and physically pertinent question ishow is the energy (10) modified by the uniformly suspended QDs in the dilute limit? Following the elegant homogenization framework developed in Ref. [37], the additional doping can be described by an effective field in the dilute limit. This effective field strongly depends on the shape and anchoring conditions on the QDs but not the size of the QDs in the dilute limit (as will be made clearer below and the size will matter in the nondilute limit). We assume the QDs are spherical in shape 
as stated above and impose preferred alignment tensors on the QD surfaces $\mathbf{Q}_{v}^{g}\left(\mathbf{Q}_{v}^{c}\right)$ for QDs outside (inside the) clusters, respectively. We assume that $\mathbf{Q}_{v}^{g}$ and $\mathbf{Q}_{v}^{c}$ are constant tensors, given by

$$
\mathbf{Q}_{v}^{g}=\sqrt{\frac{3}{2}} S_{g}^{b}\left(\mathbf{n}_{g} \otimes \mathbf{n}_{g}-\frac{1}{3} I\right)
$$

and

$$
\mathbf{Q}_{v}^{c}=\sqrt{\frac{3}{2}} S_{c}^{b}\left(\mathbf{n}_{c} \otimes \mathbf{n}_{c}-\frac{1}{3} I\right)
$$

for some fixed $S_{g}^{b}, S_{c}^{b}>0$. There is no clear argument for the choice of $S_{g}^{b}$ and $S_{c}^{b}$ in this model, but we make reasonable choices below. Furthermore, we assume that $\mathbf{n}_{g}\left(\mathbf{n}_{c}\right)$ is the same for $\mathbf{Q}_{g}$ and $\mathbf{Q}_{v}^{g}$ (likewise for $\mathbf{Q}_{c}$ and $\mathbf{Q}_{v}^{c}$ ) so that there is no director distortion at the QD interfaces. Assuming a Rapini-Papoular surface anchoring energy on the QD surfaces, the QD surface energy density is given by

$$
f_{s}^{g}\left(S_{g}, S_{c}, S_{g}^{b}, S_{c}^{b}\right)=W_{g}^{0}\left|S_{g}-S_{g}^{b}\right|^{2}+W_{c}^{0}\left|S_{c}-S_{c}^{b}\right|^{2},
$$

where $W_{g}^{0}$ and $W_{c}^{0}$ are the anchoring coefficients on the QDGS interfaces, QD-ES interfaces, respectively. For relatively strong anchoring on the QD interfaces, $W_{c}^{0}$ and $W_{g}^{0}$ can be taken to be approximately $1 \times 10^{-2} \mathrm{~J} / \mathrm{m}^{2}$ [58]. In particular, $W_{c}^{0}$ is zero for QDs outside the clusters, and $W_{g}^{0}$ is zero for QDs inside the clusters. Next, we follow the paradigm in Ref. [37] to describe the collective effects of a uniform dilute suspension of QDs with surface energies as in (13), in terms of an additional homogenized effective term in the free energy (10).

As in Ref. [34], we let $A=\left(1-a_{x}\right) a_{g}\left(T-T^{*}\right), B=(1-$ $\left.a_{x}\right) B_{g}, C=\left(1-a_{x}\right) C_{g}, D=a_{x}\left(1-a_{x}\right) \gamma / N_{c}, E=\left(1-a_{x}\right)$ $E_{\mathrm{el}}, M=\alpha_{c} a_{x} / N_{c}, N=\beta_{c} a_{x} / N_{c}, P=J E_{\mathrm{el}} a_{x}, W_{g}=(1-$ $\left.a_{x}\right) W_{g}^{0}$ and $W_{c}=\frac{a_{x}}{N_{c}} W_{c}^{0}$, where $a_{x}$ is the fixed mole fraction of the ES molecules. Moreover, we assume the $E_{\mathrm{el}}=0$ throughout this paper. In agreement with the parameter values used in Ref. [34], we use fixed values of $K_{g}=K_{c}=K=15 \mathrm{pN}, a_{g}=0.04, B_{g}=1.7, C_{g}=4.5$, $\alpha_{c}=0.22, \beta_{c}=4.0\left(\alpha_{g}, B_{g}, C_{g}, \alpha_{c}\right.$, and $\beta_{c}$ in $10^{6} / 4$ Système International units).

Following the methods in Ref. [37], we describe the dilute QD-doped BLC system by means of the total free energy below without rigorous justification but rather as a phenomenological model to describe salient features of novel nanocomposites,

$$
\begin{aligned}
\mathcal{F}= & \int\left(\frac{A}{2} S_{g}^{2}-\frac{B}{3} S_{g}^{3}+\frac{C}{4} S_{g}^{4}\right) \\
& +\left(-D S_{g} S_{c}+\frac{M}{2} S_{c}^{2}+\frac{N}{4} S_{c}^{4}\right)+K_{g}\left|\nabla S_{g}\right|^{2}+K_{c}\left|\nabla S_{c}\right|^{2} d \mathbf{x} \\
& +\epsilon^{3-2 \alpha} \int_{\partial \mathcal{P}} W_{g}\left|S_{g}-S_{g}^{b}\right|^{2} d S+\epsilon^{3-2 \alpha} \int_{\partial \mathcal{P}} W_{c}\left|S_{c}-S_{c}^{b}\right|^{2} d S,
\end{aligned}
$$

where $\mathcal{P}$ is the collection of the QDs in the suspension and $1<\alpha<\frac{3}{2}$ so that $\epsilon^{3-2 \alpha} \rightarrow 0$ as $\epsilon \rightarrow 0$. The prefactor of $\epsilon^{3-2 \alpha}$ is specific to dilute systems. The main novelty is the surface energy term originating from the QD-GS and QD-ES interfaces, and the homogenized effective field is derived in the $\epsilon \rightarrow 0$ limit as will be discussed below.

We nondimensionalize the free-energy (14) by letting

$\overline{\mathbf{x}}=\mathbf{x} / x_{s}, \overline{S_{g}}=\sqrt{\frac{27 C^{2}}{12 B^{2}}} S_{g}, \overline{S_{c}}=\sqrt{\frac{27 C^{2}}{12 B^{2}}} S_{c}, \overline{\mathcal{F}}=\frac{27^{2} C^{3}}{72 B^{4} x_{s}^{3}} \mathcal{F}$, dropping all bars for convenience (so that $S_{g}$ and $S_{b}$ denote the scaled order parameters), the dimensionless energy is (we take $E_{\mathrm{el}}=0$ ),

$$
\begin{aligned}
\mathcal{F}= & \int_{\Omega_{\epsilon}}\left(\frac{t}{2} S_{g}^{2}-S_{g}^{3}+\frac{1}{2} S_{g}^{4}\right)+\left(-C_{1} S_{g} S_{c}+C_{2} S_{c}^{2}+C_{3} S_{c}^{4}\right) \\
& +\kappa_{g}\left(\frac{d S_{g}}{d x}\right)^{2}+\kappa_{c}\left(\frac{d S_{c}}{d x}\right)^{2} d \mathbf{x}+\epsilon^{3-2 \alpha} \int_{\partial \mathcal{P}} w_{g}\left|S_{g}-S_{g}^{b}\right|^{2} \\
& \times d S+\epsilon^{3-2 \alpha} \int_{\partial \mathcal{P}} w_{c}\left|S_{c}-S_{c}^{b}\right|^{2} d S,
\end{aligned}
$$

where $\Omega_{\epsilon}$ is the three-dimensional planar cell with the QDs removed, $\mathcal{P}$ is the collection of the three-dimensional spherical QDs with the rescaled radius $\epsilon^{\alpha}$ for $1<\alpha<\frac{3}{2}$ (see the definition of $\epsilon$ above), and

$$
\begin{aligned}
t & =\frac{27 A C}{6 B^{2}}, \quad C_{1}=\frac{27 C D}{6 B^{2}}, \quad C_{2}=\frac{27 C M}{12 B^{2}}, \quad C_{3}=\frac{N}{2 C}, \\
\kappa_{g} & =\frac{27 C K_{g}}{6 B^{2} x_{s}^{2}}, \quad \kappa_{c}=\frac{27 C K_{c}}{6 B^{2} x_{s}^{2}} \\
w_{g} & =\frac{27 C W_{g}}{6 B^{2} x_{s}} \quad w_{c}=\frac{27 C W_{c}}{6 B^{2} x_{s}} .
\end{aligned}
$$

Note that $\left|\nabla S_{g}\right|^{2}=\left(\frac{d S_{g}}{d x}\right)^{2}$ since we assume that structural variations in $S_{g}$ and $S_{c}$ only occur across the cell height, $0 \leqslant x \leqslant 100$ (recall the choice of $x_{s}$ above).

In Ref. [37], the authors study minimizers of free energies of the form

$$
\iiint_{\Omega_{\epsilon}} f_{\mathrm{el}}(\boldsymbol{\nabla} \mathbf{Q})+f_{b}(\mathbf{Q}) d V+\epsilon^{3-2 \alpha} \iint_{\partial \mathcal{P}} f_{s}(\mathbf{Q}, v) d A,
$$

with $1<\alpha<\frac{3}{2}$, where $f_{\mathrm{el}}(\boldsymbol{\nabla} \mathbf{Q})$ is a general convex function of the gradient of an arbitrary order parameter $\mathbf{Q}, f_{b}$ is a polynomial function of the scalar invariants of $\mathbf{Q}$, and $f_{s}$ 's are arbitrary surface energies on the QD interfaces. The dilute limit is described by the $\epsilon \rightarrow 0$ limit, and minimizers of (17) converge to minimizers of the following homogenized energy as $\epsilon \rightarrow 0$ :

$$
\mathcal{F}_{h}(\mathbf{Q})=\int_{\Omega} f_{\mathrm{el}}(\boldsymbol{\nabla} \mathbf{Q})+f_{b}(\mathbf{Q})+f_{\text {hom }}(\mathbf{Q}) d V,
$$

where $f_{\text {hom }}=\int_{\partial \omega} f_{s}(\mathbf{Q}, v) d S, \omega$ is a representative QD and $v$ is the outward normal to $\partial \omega$. In particular, the shape, anchoring conditions, and material properties including encapsulation properties of the QD inclusions are absorbed in the definition of $f_{\text {hom }}$. The distortion effects around the QDs are also described by $f_{\text {hom }}$ for dilute systems. In our case, the QDs are spherical inclusions and applying the results in Ref. [37], we have $f_{\text {hom }}=\int_{\partial B(\mathbf{0}, 1)} f_{s}(\mathbf{Q}, v) d A, B(\mathbf{0}, 1) \subset \mathbb{R}^{3}$ is a generic three-dimensional unit ball, and $f_{s}$ is the surface energy (13). We apply this result to calculate the homogenized potential corresponding to (13), see below,

$$
f_{\text {hom }}\left(S_{g}, S_{c}\right)=w_{g}^{(1)} S_{g}^{2}-w_{g}^{(2)} S_{g}+w_{c}^{(1)} S_{c}^{2}-w_{c}^{(2)} S_{c},
$$


where

$$
\omega_{g}^{(1)}=4 \pi w_{g}, \quad \omega_{g}^{(2)}=8 \pi S_{g}^{b} w_{g}
$$

and

$$
w_{c}^{(1)}=4 \pi w_{c}, \quad w_{c}^{(2)}=8 \pi S_{c}^{b} w_{c} .
$$

Hence, the total nondimensionalized homogenized free energy is given by

$$
\begin{aligned}
\mathcal{F}= & \int_{\Omega}\left[\left(\frac{t}{2}+w_{g}^{(1)}\right) S_{g}^{2}-\sqrt{6} S_{g}^{3}+\frac{1}{2} S_{g}^{4}\right] \\
& +\left[-C_{1} S_{g} S_{c}+\left(C_{2}+w_{c}^{(1)}\right) S_{c}^{2}+C_{3} S_{c}^{4}\right] \\
& +\kappa_{g}\left(\frac{d S_{g}}{d x}\right)^{2}+\kappa_{c}\left(\frac{d S_{c}}{d x}\right)^{2}-w_{g}^{(2)} S_{g}-w_{c}^{(2)} S_{c} d \mathbf{x} .
\end{aligned}
$$

For the parameter values as stated before, we have $C_{1}=$ $0.0700692, C_{2}=0.0017$, and $C_{3}=0.0040$.

Then the equilibrium or physically observable $\left(S_{g}, S_{c}\right)$ profiles are solutions of the Euler-Lagrange equations corresponding to (22),

$$
\begin{aligned}
& \kappa_{g} \frac{d^{2} S_{g}}{d x^{2}}=2 S_{g}^{3}-3 \sqrt{6} S_{g}^{2}+\left(t+w_{g}^{(1)}\right) S_{g}-C_{1} S_{c}-w_{g}^{(1)} \\
& \kappa_{c} \frac{d^{2} S_{c}}{d x^{2}}=4 C_{3} S_{c}^{3}+\left(2 C_{2}+2 w_{c}^{(1)}\right) S_{c}-C_{1} S_{g}-w_{c}^{(2)}
\end{aligned}
$$

These equations need to be complemented by boundary conditions for $S_{g}$ and $S_{c}$, and we fix Dirichlet boundary conditions for the scalar order parameters on the bottom $(x=0)$ and top $(x=100)$ of the planar cell, i.e.,

$$
S_{g}=\frac{3+\sqrt{9-8 t}}{4}, \quad S_{c}=0 \text { on } x=0 \text { and } x=100,
$$

which corresponds to the absence of clusters on the planar cell boundaries. The boundary conditions (24) are not special, and we believe that our qualitative conclusions would hold for other choices of the Dirichlet boundary conditions too. We assume that $\mathbf{n}_{g}$ and $\mathbf{n}_{c}$ are constant unit vectors, and our analysis is independent of the choice of $\mathbf{n}_{g}$ and $\mathbf{n}_{c}$, provided they are constant vectors. We also need to specify $S_{g}^{b}$ and $S_{c}^{b}$ to determine $\omega_{g}^{(2)}$ and $w_{c}^{(2)}$ above, and we choose

$$
S_{g}^{b}=\frac{3+\sqrt{9-8 t}}{4}, \quad S_{c}^{b}=0,
$$

with $W_{g}=W_{c}=W$.

Next, we numerically solve the coupled equations in (23) to compute the equilibrium profiles of $\left(S_{g}, S_{c}\right)$ as a function of temperature and different values of $W$. The parameters $N_{c}$ and $\gamma$ are coupled, i.e., larger clusters are likely to have larger values of $N_{c}$ and $\gamma$. We expect $N_{c}$ and $\gamma$ to be smaller for the doped system compared to its undoped counterpart, based on the experimental results that suggest smaller cybotactic clusters in QD-doped BLCs compared to their undoped counterparts. We define the bulk mean order parameter $S_{m}$, which is a weighted scalar order parameter as shown below,

$$
S_{m}=\left(1-a_{x}\right) S_{g}+a_{x} S_{c} .
$$

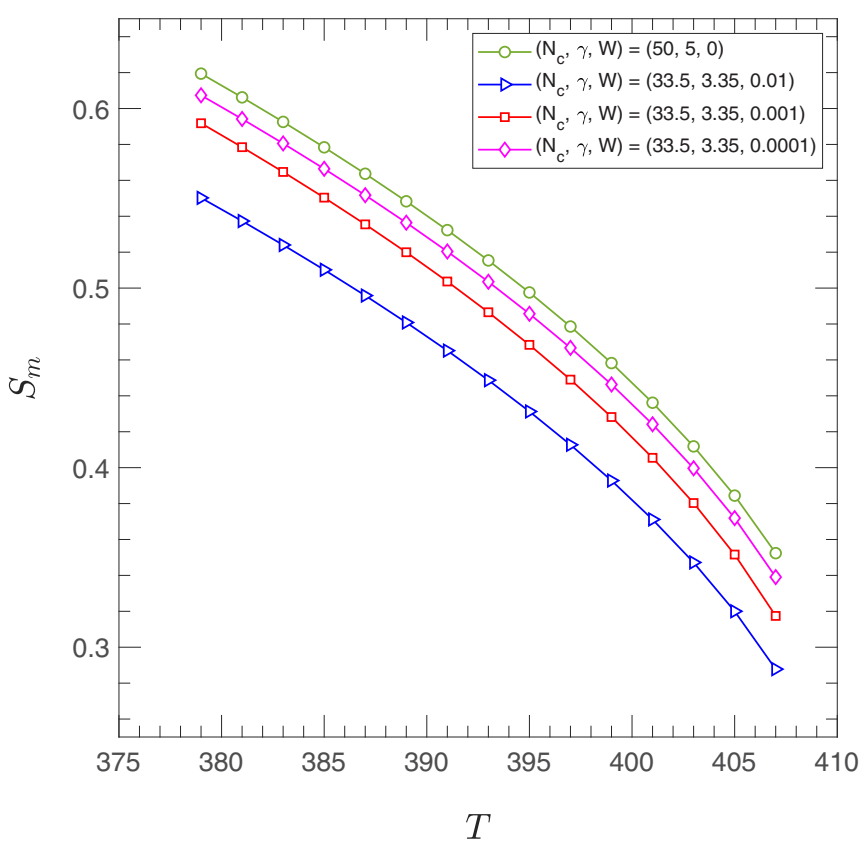

FIG. 11. Bulk mean order parameter as a function of temperature for the undoped (green circle) and the doped (red square, blue triangle, and purple diamond) cases (temperature $T$ is in kelvins).

The weighted scalar order parameter $S_{m}$ is the theoretical analog of the measured order parameters from experimental birefringence measurements. We use the value of $S_{m}$ at room-temperature $(293 \mathrm{~K})$ with $\left(N_{c}, W, \gamma\right)=(50,0,5)$ to normalize $S_{m}$. Recall that $N_{c}=50$ and $\gamma=5$ have been used to study the undoped BLC system in Ref. [34].

In Fig. 11, we plot $S_{m}$ as function of temperature for undoped and doped systems for different values of $W$. For the undoped system, $\left(N_{c}, \gamma, W\right)=(50,5,0)$ by analogy with the values used in Ref. [34]. For QD-doped systems, the experimental results suggest that the clusters are shrunk by a factor of 0.67 (qualitatively deduced by the ratio of the relaxation frequencies), and, hence, we take $N_{c}=50 \times 0.67=33.5$ and $\gamma=5 \times 0.67=3.35$ for doped systems. We plot the solution, $S_{m}$-profiles for three doped systems- $\left(N_{c}, \gamma, W\right)=$ $(33.5,3.35,0.01),\left(N_{c}, \gamma, W\right)=(33.5,3.35,0.001), \quad$ and $\left(N_{c}, \gamma, W\right)=(33.5,3.35,0.0001)$ in Fig. 11.

It is clear that this simple model clearly captures the doping-induced reduction in the values of $S_{m}$, consistent with the experimental results in Fig. 4. $S_{m}$ also decreases with increasing $T$ as expected. The numerically computed values of $S_{m}$ for the doped systems in Fig. 11 are lower than the experimentally reported values in Fig. 4, and we think an exact fitting between the numerical and the experimental results is unlikely at this stage. Furthermore, the numerical results are very sensitive to the values of the anchoring coefficient, and we simply do not have reliable estimates of the anchoring coefficients for QDs. In fact, the numerically computed $S_{m}$ 's, if fitted to the experimental data, could provide an ingenious method for estimating the surface energy coefficients for QDdoped BLC systems. It is clear that the predictions of the doped model approach the predictions of the undoped model as $W \rightarrow 0$ as expected. We do not pursue this further in this paper. 
This simple model provides a clear explanation of why the QD doping reduces $S_{m}$ in experiments-the interaction or anchoring between the QDs and the host BLC matrix increases the effective temperature [the coefficient of $S_{c}^{2}$ and $S_{g}^{2}$ in (21)] of both the GS state and the cybotactic clusters (ES state). Hence, at a given temperature, the QD-doped system experiences a shifted higher temperature and the shift is directly determined by the homogenized potential $f_{\text {hom }}$, which, in turn, is determined by the anchoring and shape of the dispersed QDs. This QD-induced effective temperature shift necessarily reduces $S_{m}$ compared to its undoped counterpart. A dopinginduced reduced $S_{m}$ qualitatively explains the experimentally observed reduced dielectric anisotropy and birefringence in doped systems, compared to undoped systems. However, several open questions remain, particularly, as to how we ascribe values to the cluster parameters $N_{c}$ and $\gamma$ and how to describe the effects of QD doping on these cluster parameters. Nevertheless, this is an attempt to mathematically model dilute suspensions of QDs in the nematic phase of BLC materials with cybotactic clusters providing qualitative agreement with experiments.

\section{CONCLUSION}

We perform experimental and theoretical studies of a QDsdispersed BLC 14-2M-CH $\mathrm{CH}_{3}$ inside a planar cell. We believe that QDs are attractive nanoinclusions for stable suspensions without aggregation effects. We present experimental optical profiles for the pristine LC and the QDs incorporated LC nanocomposite systems, tracking the textural color changes with temperature. We perform experimental measurements of the dielectric permittivity, including the dielectric dispersion and absorption spectra, and use fitting algorithms to calculate relaxation frequencies and dielectric strengths that are used to validate the existence of cybotactic clusters in the doped and undoped systems, the reduction of cluster sizes in doped systems, and the corresponding increase in activation energies. We also present experimental measurements of the optical birefringence and the orientational order parameters of the doped and undoped systems. All the experiments demonstrate doping-induced reduction of orientational order and cluster sizes that manifest in doping-induced reduced birefringence and a reduced dielectric anisotropy (qualitatively) at a fixed temperature. In terms of future experiments, we would like to investigate biaxiality in these QD-dispersed BLC systems, chirality, and prototype devices based on such simple planar cell geometries. For example, we could treat the planar cell to have conflicting boundary conditions on both the cell surfaces, naturally inducing inhomogeneous director profiles.

We support some of our experimental findings with a homogenized Landau-de Gennes-type model for a doped BLC system with two scalar order parameters $S_{g}$ and $S_{c}$ and constant director profiles. In particular, we capture the dopinginduced reduction in the mean scalar order parameter which is an informative and illuminating first step. The theory can be embellished in many ways to make it physically realistic, e.g., elastic anisotropy involving additional terms in the elastic energy density, nonconstant director profiles captured by nonconstant $\mathbf{n}_{g}$ and $\mathbf{n}_{c}$, and understanding how the QDs affect the cybotactic clusters. This could be performed by using a general two-tensor model $\mathbf{Q}_{g}$ and $\mathbf{Q}_{c}$ without making any additional assumptions about uniaxial symmetry or constant directors as in our mathematical model in this paper. However, it will be a challenge to describe the cybotactic cluster-mediated coupling between $\mathbf{Q}_{g}$ and $\mathbf{Q}_{c}$ without these restrictive assumptions, and some of these directions will be pursued in future work.

\section{ACKNOWLEDGMENTS}

We would like to thank Prof. N. V. S. Rao, Department of Chemistry, Assam University, Assam, India and Dr. G. Mohiuddin, Xi' an Jiaotong University, Xi'an, China for providing the liquid crystal samples. We thank Dr. G. Canevari for helpful discussions on the homogenized potentials. We also thank A. Sharma and I. Dierking for useful feedback on the experimental sections. A.M. thanks the University of Strathclyde for a Global Engagements Travel Grant, and A.M. thanks G. Mehl and S. Klein for helpful discussions. S.P. thanks Dr. S. Chakraborty for useful discussions on fitting of the experimental data. S.P. acknowledges IIT Delhi for financial support under a full-time Institute Assistantship. We would like to thank DST-UKIERI for generous funding to support the 3-yr Collaborative Project.

S.P. and A.S. conducted the experiments and analyzed the experimental results. Y.W. and A.M. performed the modeling and comparisons between experiments and modeling.
[1] C. Tschierske and D. J. Photinos, Biaxial nematic phases, J. Mater. Chem. 20, 4263 (2010).

[2] H. Takezoe and Y. Takanishi, Bent-core liquid crystals: their mysterious and attractive world, Jpn. J. Appl. Phys. 45, 597 (2006).

[3] A. Jákli, Liquid crystals of the twenty-first century-nematic phase of bent-core molecules, Liq. Cryst. Rev. 1, 65 (2013).

[4] O. Francescangeli, F. Vita, and E. T. Samulski, The cybotactic nematic phase of bent-core mesogens: State of the art and future developments, Soft Matter 10, 7685 (2014).

[5] V. Punjani, G. Mohiuddin, S. Kaur, R. K. Khan, S. Ghosh, and S. K. Pal, Observation of polar order and thermochromic behaviour in a chiral bent-core system exhibiting exotic mesophases due to superstructural frustration, Chem. Commun. 54, 3452 (2018).

[6] C. Keith, A. Lehmann, U. Baumeister, M. Prehm, and C. Tschierske, Nematic phases of bent-core mesogens, Soft Matter 6, 1704 (2010).

[7] M. Cestari, S. Diez-Berart, D. A. Dunmur, A. Ferrarini, M. R. de la Fuente, D. J. B. Jackson, D. O. Lopez, G. R. Luckhurst, M. A. Perez-Jubindo, R. M. Richardson, J. Salud, B. A. Timimi, and H. Zimmermann, Phase behavior and properties of the liquid-crystal dimer $1^{\prime \prime}, 7^{\prime \prime}$-bis(4-cyanobiphenyl-4'-yl) heptane: A twist-bend nematic liquid crystal, Phys. Rev. E 84, 031704 (2011). 
[8] V. Borshch, Y.-K. Kim, J. Xiang, M. Gao, A. Jákli, V. P. Panov, J. K. Vij, C. T. Imrie, M.-G. Tamba, G. H. Mehl et al., Nematic twist-bend phase with nanoscale modulation of molecular orientation, Nat. Commun. 4, 2635 (2013).

[9] S. Taushanoff, K. Van Le, J. Williams, R. J. Twieg, B. Sadashiva, H. Takezoe, and A. Jákli, Stable amorphous blue phase of bent-core nematic liquid crystals doped with a chiral material, J. Mater. Chem. 20, 5893 (2010).

[10] G. Shanker, M. Prehm, M. Nagaraj, J. K. Vij, M. Weyland, A. Eremin, and C. Tschierske, 1, 2, 4-oxadiazole-based bent-core liquid crystals with cybotactic nematic phases, ChemPhysChem 15, 1323 (2014).

[11] G. Shanker, M. Nagaraj, A. Kocot, J. K. Vij, M. Prehm, and C. Tschierske, Nematic phases in 1, 2, 4-oxadiazole-based bentcore liquid crystals: is there a ferroelectric switching? Adv. Funct. Mater. 22, 1671 (2012).

[12] S. Ghosh, N. Begum, S. Turlapati, S. K. Roy, A. K. Das, and N. V. Rao, Ferroelectric-like switching in the nematic phase of four-ring bent-core liquid crystals, J. Mater. Chem. C 2, 425 (2014).

[13] O. Francescangeli, V. Stanic, S. I. Torgova, A. Strigazzi, N. Scaramuzza, C. Ferrero, I. P. Dolbnya, T. M. Weiss, R. Berardi, L. Muccioli et al., Ferroelectric response and induced biaxiality in the nematic phase of bent-core mesogens, Adv. Funct. Mater. 19, 2592 (2009).

[14] L. Marino, A. T. Ionescu, S. Marino, and N. Scaramuzza, Dielectric investigations on a bent-core liquid crystal, J. Appl. Phys. 112, 114113 (2012).

[15] C. Bailey, K. Fodor-Csorba, J. T. Gleeson, S. N. Sprunt, and A. Jákli, Rheological properties of bent-core liquid crystals, Soft Matter 5, 3618 (2009).

[16] S. Kashima, M. Chiba, Y. Takanishi, J. Yamamoto, and A. Yoshizawa, Polar order of an achiral taper-shaped liquid crystal in the uniaxial smectic a phase, J. Mater. Chem. C 6, 5521 (2018).

[17] M. Alaasar, S. Poppe, C. Kerzig, C. Klopp, A. Eremin, and C. Tschierske, Cluster phases of 4-cyanoresorcinol derived hockey-stick liquid crystals, J. Mater. Chem. C 5, 8454 (2017).

[18] S. H. Hong, R. Verduzco, J. T. Gleeson, S. Sprunt, and A. Jákli, Nanostructures of liquid crystal phases in mixtures of bent-core and rod-shaped molecules, Phys. Rev. E 83, 061702 (2011).

[19] V. Domenici, Dynamics in the isotropic and nematic phases of bent-core liquid crystals: Nmr perspectives, Soft Matter 7, 1589 (2011).

[20] V. Görtz, C. Southern, N. W. Roberts, H. F. Gleeson, and J. W. Goodby, Unusual properties of a bent-core liquid-crystalline fluid, Soft Matter 5, 463 (2009).

[21] F. Haraguchi, K.-i. Inoue, N. Toshima, S. Kobayashi, and K. Takatoh, Reduction of the threshold voltages of nematic liquid crystal electrooptical devices by doping inorganic nanoparticles, Jpn. J. Appl. Phys. 46, L796 (2007).

[22] H.-Y. Chen, W. Lee, and N. A. Clark, Faster electro-optical response characteristics of a carbon-nanotube-nematic suspension, Appl. Phys. Lett. 90, 033510 (2007).

[23] W. Lee, C.-Y. Wang, and Y.-C. Shih, Effects of carbon nanosolids on the electro-optical properties of a twisted nematic liquid-crystal host, Appl. Phys. Lett. 85, 513 (2004).

[24] R. Khan, S. Turlapati, N. Rao, and S. Ghosh, Sign-inversion of elastic anisotropy in a bent-core nematic liquid crystal doped with carbon nanodots, J. Mol. Liq. 225, 328 (2017).
[25] J. Kumar, V. Prasad, and M. Manjunath, Quantum dots dispersed hockey stick nematic liquid crystal: Studies on dielectric permittivity, elastic constants and electrical conductivity, J. Mol. Liq. 266, 10 (2018).

[26] U. Shivakumar, J. Mirzaei, X. Feng, A. Sharma, P. Moreira, and T. Hegmann, Nanoparticles: complex and multifaceted additives for liquid crystals, Liq. Cryst. 38, 1495 (2011).

[27] S. Orlandi, E. Benini, I. Miglioli, D. R. Evans, V. Reshetnyak, and C. Zannoni, Doping liquid crystals with nanoparticles. a computer simulation of the effects of nanoparticle shape, Phys. Chem. Chem. Phys. 18, 2428 (2016).

[28] J. Mirzaei, M. Urbanski, K. Yu, H.-S. Kitzerow, and T. Hegmann, Nanocomposites of a nematic liquid crystal doped with magic-sized cdse quantum dots, J. Mater. Chem. 21, 12710 (2011).

[29] B. Kinkead and T. Hegmann, Effects of size, capping agent, and concentration of cdse and cdte quantum dots doped into a nematic liquid crystal on the optical and electro-optic properties of the final colloidal liquid crystal mixture, J. Mater. Chem. 20, 448 (2010).

[30] J. Mirzaei, M. Reznikov, and T. Hegmann, Quantum dots as liquid crystal dopants, J. Mater. Chem. 22, 22350 (2012).

[31] T. Zhang, C. Zhong, and J. Xu, Cds-nanoparticle-doped liquid crystal displays showing low threshold voltage, Jpn. J. Appl. Phys. 48, 055002 (2009).

[32] M. Urbanski, J. Mirzaei, T. Hegmann, and H.-S. Kitzerow, Nanoparticle doping in nematic liquid crystals: distinction between surface and bulk effects by numerical simulations, ChemPhysChem 15, 1395 (2014).

[33] W. Maier and A. Saupe, A simple molecular theory of the nematic liquid-crystalline state, Dynamics and Defects in Liquid Crystals: A Festschrift in Honor of Alfred Saupe (CRC Press, 1998), p. 389.

[34] S. Patranabish, Y. Wang, A. Sinha, and A. Majumdar, Onedimensional theoretical analysis of coupling and confinement effects on the cybotactic clusters of bent-core nematic liquid crystals, Phys. Rev. E 99, 012703 (2019).

[35] N. V. Madhusudana, Two-state model for nematic liquid crystals made of bent-core molecules, Phys. Rev. E 96, 022710 (2017).

[36] S. Patranabish, G. Mohiuddin, N. Begum, A. R. Laskar, S. K. Pal, N. V. Rao, and A. Sinha, Cybotactic nematic phase of achiral unsymmetrical bent-core liquid crystals-quelling of polar ordering and the influence of terminal substituent moiety, J. Mol. Liq. 257, 144 (2018).

[37] G. Canevari and A. Zarnescu, Design of effective bulk potentials for nematic liquid crystals via colloidal homogenisation, Math. Models Methods Appl. Sci. 30, 309 (2020).

[38] P. Kumar, S. Debnath, N. V. Rao, and A. Sinha, Nanodoping: a route for enhancing electro-optic performance of bent core nematic system, J. Phys.: Condens. Matter 30, 095101 (2018).

[39] A. Chakraborty, S. Chakraborty, and M. K. Das, Effect of hockey-stick-shaped molecules on the critical behavior at the nematic to isotropic and smectic-a to nematic phase transitions in octylcyanobiphenyl, Phys. Rev. E 91, 032503 (2015).

[40] S. Chakraborty, M. K. Das, A. Bubnov, W. Weissflog, D. Wegłowska, and R. Dabrowski, Induced frustrated twist grain boundary liquid crystalline phases in binary mixtures of achiral hockey stick-shaped and chiral rod-like materials, J. Mater. Chem. C 7, 10530 (2019). 
[41] R. Hoffman and M. W. Davidson, Michel-Levy birefringence chart, https://www.olympus-lifescience.com/en/microscoperesource/primer/techniques/polarized/michel/.

[42] A. Nafees, G. Kalita, M. K. Paul, A. Sinha, and N. V. Rao, Effect of methoxy group instead of polar group in the nematic phase of four-ring bent-core liquid crystals, RSC Adv. 5, 7001 (2015).

[43] I. Dierking, Textures of Liquid Crystals (Wiley-VCH, Weinheim, 2003).

[44] I. Chirtoc, M. Chirtoc, C. Glorieux, and J. Thoen, Determination of the order parameter and its critical exponent for $n \mathrm{cb}(n$ $=5-8$ ) liquid crystals from refractive index data, Liq. Cryst. 31, 229 (2004).

[45] I. Haller, Thermodynamic and static properties of liquid crystals, Prog. Solid State Chem. 10, 103 (1975).

[46] W. Haase and S. Wróbel, in Relaxation Phenomena: Liquid Crystals, Magnetic Systems, Polymers, High-Tc Superconductors, Metallic Glasses, edited by W. Haase and S. Wróbel (Springer-Verlag, Berlin-Heidelberg, 2003).

[47] C. Zhang, M. Gao, N. Diorio, W. Weissflog, U. Baumeister, S. Sprunt, J. T. Gleeson, and A. Jákli, Direct Observation of Smectic Layers in Thermotropic Liquid Crystals, Phys. Rev. Lett. 109, 107802 (2012).

[48] Y. P. Panarin, S. P. Sreenilayam, J. K. Vij, A. Lehmann, and C. Tschierske, Formation and development of nanometer-sized cybotactic clusters in bent-core nematic liquid crystalline compounds, Beilstein J. Nanotechnol. 9, 1288 (2018).

[49] D. Wiant, S. Stojadinovic, K. Neupane, S. Sharma, K. FodorCsorba, A. Jákli, J. T. Gleeson, and S. Sprunt, Critical behavior at the isotropic-to-nematic phase transition in a bent-core liquid crystal, Phys. Rev. E 73, 030703(R) (2006).
[50] P. Tadapatri, U. S. Hiremath, C. Yelamaggad, and K. Krishnamurthy, Permittivity, conductivity, elasticity, and viscosity measurements in the nematic phase of a bent-core liquid crystal, J. Phys. Chem. B 114, 1745 (2010).

[51] R. Douali, C. Legrand, V. Faye, and H. Nguyen, Dielectric dispersion in the $\mathrm{s}^{*} \mathrm{c} \alpha$ phase of an antiferroelectric liquid crystal, Mol. Cryst. Liq. Cryst. Sci. Technol., Sect. A 328, 209 (1999).

[52] S. Havriliak and S. Negami, A complex plane analysis of $\alpha$ dispersions in some polymer systems, J. Polym. Sci. C 14, 99 (1966).

[53] S. Havriliak and S. Negami, A complex plane representation of dielectric and mechanical relaxation processes in some polymers, Polymer 8, 161 (1967).

[54] P. Nayek, S. Ghosh, S. Roy, T. P. Majumder, and R. Dabrowski, Electro-optic and dielectric investigations of a perfluorinated compound showing orthoconic antiferroelectric liquid crystal, J. Mol. Liq. 175, 91 (2012).

[55] S. Ghosh, P. Nayek, S. K. Roy, T. P. Majumder, and R. Dabrowski, Dielectric relaxation spectroscopy and electrooptical studies of a new, partially fluorinated orthoconic antiferroelectric liquid crystal material exhibiting v-shaped switching, Liq. Cryst. 37, 369 (2010).

[56] S. K. Saha, G. Mohiuddin, M. K. Paul, S. P. Gupta, R. K. Khan, S. Ghosh, and S. K. Pal, Polar switching and cybotactic nematic ordering in 1, 3, 4-thiadiazole-based short-core hockey stickshaped fluorescent liquid crystals, ACS Omega 4, 7711 (2019).

[57] P. Salamon, N. Éber, Á. Buka, J. T. Gleeson, S. Sprunt, and A. Jákli, Dielectric properties of mixtures of a bent-core and a calamitic liquid crystal, Phys. Rev. E 81, 031711 (2010).

[58] M. Ravnik and S. Žumer, Landau-de gennes modelling of nematic liquid crystal colloids, Liq. Cryst. 36, 1201 (2009). 